\title{
Artificial Intelligence-Based Colorectal Polyp Histology Prediction: High Accuracy in Larger Polyps
}

\author{
Naoki Muguruma and Tetsuji Takayama \\ Department of Gastroenterology and Oncology, Tokushima University Graduate School of Biomedical Sciences, Tokushima, Japan
}

See “Artificial Intelligence-Based Colorectal Polyp Histology Prediction by Using Narrow-Band Image-Magnifying Colonoscopy" by Istvan Racz, Andras Horvath, Noemi Kranitz, et al., on page 113-121. Clin Endosc 2022;55:45-46

Artificial intelligence (AI) has recently become an essential technology applied to various fields, including weather forecast, social networking, automatic driving, and video games. ${ }^{1}$ This evolution has also rapidly affected medicine and healthcare, starting with the dermatological classification of skin cancer with deep neural networks. ${ }^{2}$ In the field of gastrointestinal endoscopy, computer-aided diagnosis (CAD) is gaining increased attention as a reliable technological revolution that can not only compensate for human errors but also perform tasks that are beyond human ability., The major roles and expected advantages of CAD include computer-aided detection of lesions that are barely detectable and computer-aided histopathological diagnosis without biopsy or removal of tissue. Colorectal cancer is a major cause of cancer-related death worldwide, increasing the adenoma detection rate, one of the vital quality indicators in colonoscopy, is a topic of high priority, while optical biopsy is another tool that may help to reduce increasing medical expenses. Optical biopsy has gained more acceptance because it may be more cost-effective and

Received: November 1, 2021 Revised: November 3, 2021

Accepted: November 4, 2021

Correspondence: Naoki Muguruma

Department of Gastroenterology and Oncology, Tokushima University Graduate School of Biomedical Sciences, 3-18-15, Kuramoto-cho, Tokushima 770-8503, Japan

Tel: +81-88-633-7124, Fax: +81-88-633-9235, E-mail: muguruma.clin.med@ gmail.com

ORCID: https://orcid.org/0000-0002-4740-4809

(c) This is an Open Access article distributed under the terms of the Creative Commons Attribution Non-Commercial License (http://creativecommons.org/ licenses/by-nc/3.0) which permits unrestricted non-commercial use, distribution, and reproduction in any medium, provided the original work is properly cited. time-efficient than traditional procedures, e.g. histopathology of removed polyps, especially small non-malignant polyps, do not need to be reviewed by a pathologist. ${ }^{1}$

In an issue of Clinical Endoscopy, Racz et al. ${ }^{5}$ reported that the artificial intelligence-based polyp histology prediction (AIPHP) software could predict colorectal polyp histology with high accuracy in the larger polyp subgroup. In this study, a total of 373 colorectal polyps were removed by polypectomy or mucosectomy after detection, first by high-definition colonoscopy followed by observation with narrow-band imaging $(\mathrm{NBI})$ at the maximum $(\times 65)$ magnification. Then, the stored NBI images were analyzed according to the NBI International Colorectal (NICE) classification by three endoscopists with more than 20 years of experience, and AIPHP in a parallel fashion. They used histology as the gold standard based on the WHO classification, and two-class classifications were considered as hyperplastic or neoplastic, including sessile serrated lesions (SSL), adenomas, and invasive adenocarcinomas. The AIPHP software was based on the vascular pattern and color of the polyps, but human interaction is needed to select the area of interest because the present version cannot automatically find it. The main results are as follows: the accuracy of AIPHP was $86.6 \%$ overall, $82.1 \%$ for diminutive polyps, and $92.2 \%$ for non-diminutive polyps. The accuracy of the hyperplastic histology prediction by NICE was $95.2 \%$ in the diminutive polyps and $97.1 \%$ in all evaluated polyps, while the accuracy by the AIPHP method was $82.1 \%$ and $86.6 \%$, thus significantly better with NICE in both categories. Linear regression correlation coefficient results indicate that the AIPHP method was significantly more accurate in the bigger polyps than in the smaller polyps, when a detailed calculation was 
performed.

The results demonstrated that presently the the diagnostic performance of AIPHP did not surpass that of NICE, which is an already tested classification method by human endoscopists. Therefore, the conclusion they have drawn was relatively modest. However, this study has elucidated some latent and profound issues in developing AI technology in conjunction with knowledge and experience integrated by humans. First, the development of an AI diagnostic algorithm requires a human diagnosis and labeling to train the AI. ${ }^{1}$ The system used in this study involved human interaction in selecting good-quality areas, which may build an inherent bias into the system from the start. Second, AI will not be able to compensate for the risk of suboptimal colonoscopy in cases of inadequate cleaning that may affect the classification of the surface pattern of each polyp. ${ }^{6}$ Although smaller numbers of SSL were found in this study, this flat and isochromic polyp is frequently covered with a mucus cap or debris during routine colonoscopy, which may hinder the original character. ${ }^{7}$ Third, this system is a proprietary specification system developed in a single center, and there may be an uneven spectrum of diagnostic features, including pathology. Hence, further external validation of the accuracy of a large number of facilities are needed. Fourth, along with the evolution of endoscopic AI, high precision and negative predictive values should be warranted. ${ }^{8}$ We have to wait until multicenter, prospective randomized studies with a large number of cases have been conducted. Currently, we are only halfway through the distribution of AI; however, it will gain wider acceptance not only as a clinical tool in daily practice but also as an educational gadget in the near future.

\section{Conflicts of Interest}

The authors have no potential conflicts of interest.

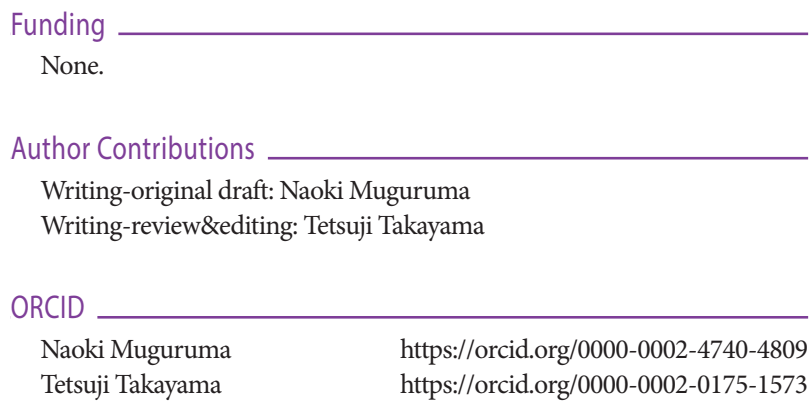

\section{REFERENCES}

1. Abadir AP, Ali MF, Karnes W, Samarasena JB. Artificial intelligence in gastrointestinal endoscopy. Clin Endosc 2020;53:132-141.

2. Esteva A, Kuprel B, Novoa RA, et al. Dermatologist-level classification of skin cancer with deep neural networks. Nature 2017;542:115-118.

3. Mori Y, Neumann H, Misawa M, Kudo SE, Bretthauer M. Artificial intelligence in colonoscopy - now on the market. What's next? J Gastroenterol Hepatol 2021;36:7-11.

4. Misawa M, Kudo SE, Mori Y, et al. Current status and future perspective on artificial intelligence for lower endoscopy. Dig Endosc 2021;33:273284.

5. Racz I, Horvath A, Kranitz N, Kiss G, Regoczi H, Horvath Z. Artificial intelligence-based colorectal polyp histology prediction by using narrow-band image-magnifying colonoscopy. Clin Endosc 2022;55:113121.

6. Milluzzo SM, Cesaro P, Grazioli LM, Olivari N, Spada C. Artificial intelligence in lower gastrointestinal endoscopy: the current status and future perspective. Clin Endosc 2021;54:329-339.

7. Okamoto K, Kitamura S, Kimura T, et al. Clinicopathological characteristics of serrated polyps as precursors to colorectal cancer: current status and management. J Gastroenterol Hepatol 2017;32:358-367.

8. Rex DK, Kahi C, O’Brien M, et al. The American Society for Gastrointestinal Endoscopy PIVI (Preservation and Incorporation of Valuable Endoscopic Innovations) on real-time endoscopic assessment of the histology of diminutive colorectal polyps. Gastrointest Endosc 2011;73:419422 